\title{
Spaceflight and modeled microgravity effects on microbial growth and virulence
}

\author{
Jason A. Rosenzweig • Ohunene Abogunde • \\ Kayama Thomas • Abidat Lawal • Y-Uyen Nguyen • \\ Ayodotun Sodipe $\cdot$ Olufisayo Jejelowo
}

Received: 24 July 2009 /Revised: 26 August 2009 /Accepted: 27 August 2009/Published online: 22 October 2009

(C) Springer-Verlag 2009

\begin{abstract}
For unsuspecting bacteria, the difference between life and death depends upon efficient and specific responses to various stressors. Facing a much larger world, microbes are invariably challenged with ever-changing environments where temperature, $\mathrm{pH}$, chemicals, and nutrients are in a constant state of flux. Only those that are able to rapidly reprogram themselves and express subsets of genes needed to overcome the stress will survive and outcompete neighboring microbes. Recently, low shear stress, emulating microgravity (MG) experienced in space, has been characterized in a number of microorganisms including fungi and prokaryotes ranging from harmless surrogate organisms to bona fide pathogens. Interestingly, $M G$ appears to induce a plethora of effects ranging from enhanced pathogenicity in several Gram-negative enterics to enhanced biofilm formation. Furthermore, MG-exposed bacteria appeared better able to handle subsequent stressors including: osmolarity, $\mathrm{pH}$, temperature, and antimicrobial challenge while yeast exhibited aberrant budding post-MG-exposure. This review will focus on MG-induced alterations of virulence in various microbes with the emphasis placed on bacteria.
\end{abstract}

Keywords Space microbiology · Microgravity - Low-shear modeled microgravity Virulence $\cdot$ Bacteria

J. A. Rosenzweig $\cdot$ O. Abogunde $\cdot$ K. Thomas $\cdot$ A. Lawal $\cdot$

Y.-U. Nguyen · A. Sodipe $\cdot$ O. Jejelowo

Department of Biology Houston, Texas Southern University, Houston, TX, USA

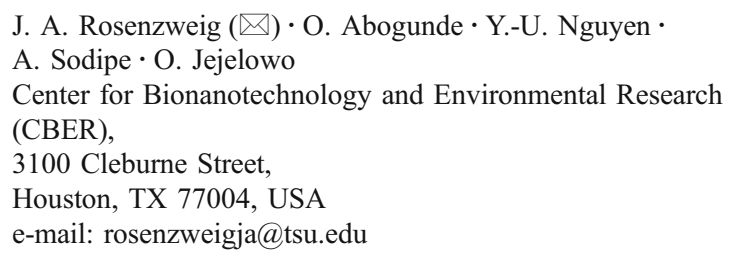

\section{Introduction}

Microbes are highly evolved and occupy unique environmental niches. In order for the plethora of microbes to persist and thrive, they must be highly responsive to changes in their local environments. Some of these environmental changes are experienced in the form of temperature, oxidative, host cell induced, and/or chemical stresses. Only those microbes which can readily reprogram themselves (in response to the stress) will be successful in staying the course and enjoying the benefits of post-stress conditions. Spaceflight poses two unique microbial spacelike stresses (SLSs): microgravity (MG) as well as other low shear environments and space irradiation from various sources. Both of the aforementioned stresses could be experienced by either normal flora microbes (i.e., resident flora present on astronauts) or by exogenous and sometimes unavoidable sources of contamination ferried beyond the Earth's atmosphere during spaceflight. One of the potential fears is that through SLS-induced responses, resident flora could become opportunistic and function more as a pathogen. Opportunistic infection coupled with compromised immune responses of space-bound astronaut hosts could have potentially life-threatening consequences. This mini-review will be primarily focused on the former SLS, MG, and other low shear environments, and the physiological and genetic changes exerted on microbes.

Recently, several important studies have demonstrated a key role for microgravity and the low fluid shear dynamics associated with microgravity in the regulation of microbial gene expression, physiology, and even pathogenesis (Nickerson et al. 2003; Nickerson et al. 2004). In fact, some studies employ MG as a means to study changes at the evolutionary levels to better understand how gravity itself has helped shaped life on our planet. 
Normal/ground gravity vs. low-shear modeled microgravity

In order to emulate MG on Earth, National Aeronautics and Space Administration (NASA) researchers developed a highaspect ratio vessel (HARV) bioreactor; HARVs as well as other rotating wall vessels (RWV) are now commercially available (Synthecon, Inc. Houston, TX). The HARV bioreactor produces an environmental condition in which the gravitational vectors are randomized over the surface of the cells. This randomization emulates a state of constant free-fall and reduction in gravity creating a sustained lowshear modeled microgravity (LSMMG) environment for cell growth. Although designed to create a LSMMG environment, the RWV can also be used to grow cells under normal gravity by simply changing the position of the bioreactor (Fig. 1).

\section{Responses of bacteria to LSMMG}

\section{Microgravity enhances Salmonella enterica serovar Typhimurium virulence}

Bacteria are able to modulate their metabolism to enhance survival during periods of environmental change. For example, Salmonella enterica serovar Typhimurium increases its virulence potential under LSMMG growth conditions. When mice were inoculated with MG-exposed salmonellae, they had a markedly reduced survival rate of 6-days post-infection. Ten days post-infection, the difference was more pronounced with only $20 \%$ of mice surviving after inoculation with MG-exposed bacteria as compared with $60 \%$ of those infected with control salmonellae (Nickerson et al. 2000). In an independent study using the antiorthostatic tail suspension (AOS) model where mice are suspended from their tails to emulate microgravity experienced by the host, the mouse mortality rate of MG-exposed salmonellae rose from $60 \%$ at day 3 post-infection to $100 \%$ mortality using a $1 \times 10^{7} \mathrm{CFU} / \mathrm{ml}$ infectious dose (Chopra et al. 2006).

Furthermore, salmonellae grown under simulated $\mathrm{MG}$, as compared with identical growth conditions at normal gravity $(\mathrm{NG})$, displayed increased resistance to environmental stresses (acid, thermal, and osmotic), increased ability to survive within macrophages, and alterations in protein levels. There was a threefold observed enhanced ability of the MG-exposed bacteria to survive as compared with the same strain grown under $\mathrm{NG}$ after exposure to acidic conditions. As it turns out, the salmonellae-enhanced survival in acidic environments was independent of RpoS, a Sigma factor involved in the regulation of known stress response genes (Wilson et al. 2002a). Interestingly, fur

\section{b}
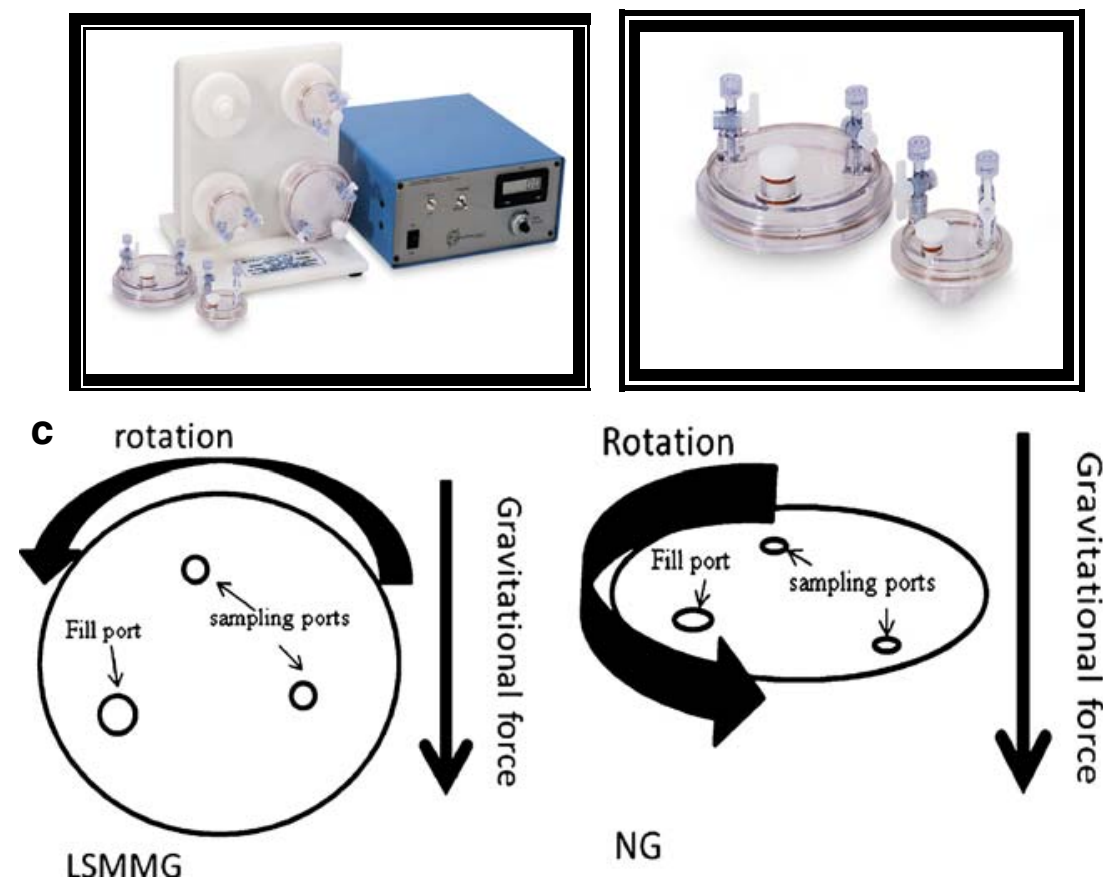

NG

Depicted are disposable HARVs (photos courtesy of Synethcon Inc.). c The operating orientations of the RWV are depicted. In the LSMMG orientation, the axis of rotation of the RWV is perpendicular to the direction of the gravity force vector. In the normal-gravity $(N G)$ orientation, the axis of rotation is parallel to the gravity force 
(which encodes the ferric iron uptake regulator Fur) and related genes $f e p D$, sufC, sufS, $f e o B$, which have all been previously shown to regulate a number of other processes besides iron metabolism in E. coli and the salmonellae (such as acid resistance), were found to be regulated by LSMMG. A number of potential Fur-binding sites were found to be located up-stream of several different LSMMGregulated genes. Fur mutants showed no detectable resistance to acid stresses induced after LSMMG exposure indicating that, unlike RpoS, Fur is required for the LSMMG-induced acid resistance in Salmonella enterica serovar Typhimurium (Wilson et al. 2002b).

Microgravity induces enhanced virulence of both enteropathogenic $E$.coli and salmonellae

In a separate global approach, two-dimensional gel electrophoresis was performed to examine the global pattern of protein expression in MG exposed EPEC and S. typhimurium. In EPEC, 74 proteins were up-regulated while 18 proteins were found to be less abundant. Furthermore, 62 produced were exclusively expressed in EPEC grown in HARVs. In contrast, S. typhimurium exhibited 35 upregulated proteins, 33 down-regulated proteins, and expression of 57 proteins exclusively expressed under simulated MG conditions. Additionally, using Northern blot analysis, expressions of the entero-toxigenic E. coli (ETEC) gene encoding the LT-1 toxin increased from eightfold to tenfold after LSMMG growth (Chopra et al. 2006).

Interestingly, LSMMG prompted adherent-invasive $E$. coli (AIEC) to become more adherent to a mammalian gastrointestinal epithelial-like cell line (Caco-2), and in the absence of the global stress regulator RpoS, AIEC host cell adherence was even more pronounced; however, no enhanced invasion was observed. Furthermore, in a manner similarly observed in the salmonellae, low shear stress did result in enhanced tolerance to acid and thermal stressors while the enhanced LSMMG-induced oxidative stress response was found to be RpoS dependent (Allen et al. 2008).

In a Salmonella enterica serovar Typhimurium microarray experiment, LSMMG also induced differential expression of various genes distributed throughout the chromosome, representing functionally diverse groups including transcriptional regulators, virulence factors, lipopolysaccharide biosynthetic enzymes, and iron-utilization enzymes. Out of approximately 4,600 genes identified in the species, 163 were found to be differentially expressed, with 97 and 68 down- and up-regulated, respectively, under LSMMG conditions. Fourteen of these genes were found to encode transcriptional regulation proteins. Interestingly, the $r f b$ operon, involved in lipopolysaccharide (LPS) side chain synthesis, was down-regulated by MG. In line with this result, it was also found that the overall level of LPS decreased under MG conditions.

Surprisingly, mRNA levels of virulence genes $(\operatorname{org} A$, $\operatorname{prg} H$, sipD, invI, invA, pigB, sseB, ssaL, ssaV, and sseJ) located in the Salmonella pathogenicity islands SPI-1 and SPI-2 encoding the two salmonellae type three secretion systems/injectisomes (TTSSs) were down-regulated by MG (Wilson et al. 2002a) despite enhanced bacterial virulence in cell culture and murine infections (Nickerson et al. 2000; Chopra et al. 2006; Wilson et al. 2008). These seemingly contradictory pieces of data suggest that MG might be influencing virulence in a TTSS-independent manner or at least by some alternative mechanism.

In another study, four independent microarray analysis software programs were used to eliminate spurious background noise and better reveal truly differentially expressed $S$. typhimurium genes under MG. Only genes that were differentially expressed using all four software programs were considered, and 22 genes were affected by MG conditions. The nine up-regulated genes included stress response genes, while the 13 down-regulated genes included various transcriptional regulators, $c p x P$ (a periplasmic repressor of the Cpx regulon encoding a two component signal transduction system), $r s e A$ which encodes (sigma 24), and cell invasion genes, such as $i n v \mathrm{~F}, \operatorname{prgH}$, $p r g \mathrm{~J}$, sic $\mathrm{P}$, and $о m p \mathrm{~W}$. Alterations of selected genes were then verified by the use of real-time polymerase chain reaction (PCR) (Chopra et al. 2006).

In another independent study, S. typhimurium flown on an actual spaceflight mission STS-115 was examined for changes at the genotypic and phenotypic level. One hundred sixty-seven genes were found to be differentially expressed, 69 of which were up-regulated and 98 were down-regulated. Also noted were the altered levels of 73 out of the 251 proteins identified via 2-dimensional protein gel analysis (Wilson et al. 2007).

Since it appeared that MG-induced global S. typhimurium transcriptional alternations, it was suspected that a global regulator might be involved in the MG-induced genetic reprogramming. Hfq is a well characterized RNA chaperone that binds to small RNA and mRNA molecules to facilitate their translation by working in conjunction with other specialized metabolic factors. During MG-induced stress, $h f q$ was one of the differentially expressed $S$. typhimurium transcripts. A $\Delta h f q$ deletion mutant strain was characterized after exposure to MG. Interestingly, under MG conditions, Hfq appeared to play a role in the acid stress survival of bacteria as well as enhance the intracellular invasion and survival of $S$. typhimurium within J774 macrophages. Upon transcriptome analysis, expression profiling revealed the up-regulation of several genes including ompA, ompC, and ompD. Hfq is known to be intimately involved in periplasmic stress signaling path- 
ways that depend on the activity levels of three key proteins: RpoE, DksA, and RseB. Interestingly, all three of the genes encoding the aforementioned proteins were differentially expressed while in flight strongly suggesting that the conserved RNA-binding regulatory protein Hfq played a role in the MG response (Wilson et al. 2007).

During the STS-115 space mission, salmoneallae were grown in two types of media either LB or a minimal medium M9 (containing greater phosphate ion concentration). Interestingly, the enhanced virulence of the MGexposed salmonellae observed while grown in LB medium was not observed if grown in the M9 minimal medium. After supplementing the LB medium with phosphate ions and flying $S$. typhimurium aboard another space mission STS-123, decreased virulence was observed (i.e., increased $\mathrm{LD}_{50}$ dose) as well as greater percentage of mouse survival (Wilson et al. 2008).

In addition to the characterization of LSMMG-exposed pathogenic E. coli (Chopra et al. 2006), E. coli MG 1655 was characterized at the transcriptome level after LSMMG exposure as well. Surprisingly, unlike what was reported earlier with the salmonellae, EPEC, and ETEC (Nickerson et al. 2000; Chopra et al. 2006; Wilson et al. 2008), there was no evidence of LSMMG-responsive genes. However, the genes of known function that were identified appeared to play a role in the cell envelope structure (Tucker et al. 2007).

Beyond cell culture infection assays, a murine infection with salmonellae grown on shuttle STS-115 orbiting the Earth displayed an earlier time of death, increased percent of mortality, and a decreased $\mathrm{LD}_{50}$ value compared with those infected with ground controls (Table 1). These data, therefore, were in agreement with studies of S. typhimurium grown under LSMMG conditions (Wilson et al. 2007) thereby validating previous Earth-bound studies of enhanced virulence using cell culture infection studies and HARV-simulated LSMMG (Chopra et al. 2006; Wilson et al. 2002b).

Microgravity co-culture of bacteria and host cells: how does the host feel?

When mouse macrophages (RAW 264.7) were infected with EPEC grown under MG conditions for $4 \mathrm{~h}$, a 2.5 -fold higher level of tumor necrosis factor $\alpha(\mathrm{TNF} \alpha)$ cytokine was observed when compared with the same macrophages infected with NG-grown EPEC (Chopra et al. 2006).

MG-grown S. typhimurium also induced changes in host protein levels. When NG-grown epithelial cells (SKOV3 ovarian carcinoma cells) were infected with MG-grown $S$. typhimurium, enhanced prostaglandin E2 $\left(\mathrm{PGE}_{2}\right)$ levels were observed at several time points tested, and at $4 \mathrm{~h}$ postinfection $9 \mathrm{ng} / \mathrm{ml}$ of $\mathrm{PGE}_{2}$ was observed compared with

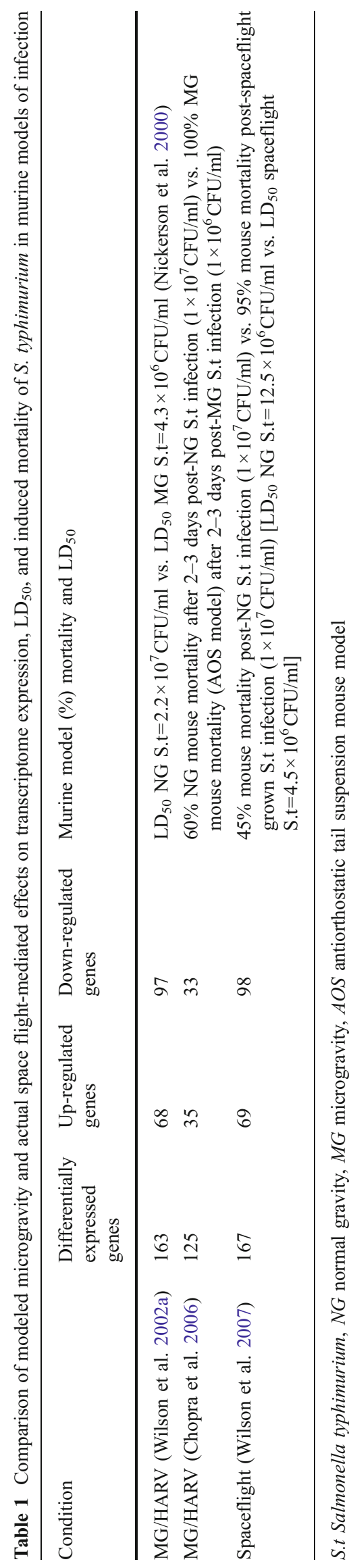


only $6.5 \mathrm{ng} / \mathrm{ml}$ made when all cells were grown in NG conditions. Interestingly, when S. typhimurium and SKOV3 were co-cultured together under MG conditions, there was a dramatic decrease $(2 \mathrm{ng} / \mathrm{ml}$ at the same 4 -h time point) in the amount of $\mathrm{PGE}_{2}$ made (Chopra et al. 2006). In stark contrast, transcript expression levels of two genes involved in $\mathrm{PGE}_{2}$ production cox-2 (which encodes cyclooxygenase) and plaa (which encodes phospholipase $\mathrm{A}_{2}$-activating protein) were found to be more abundant at later time points tested (30-180 min) in MG co-cultured S. typhimurium and SKOV3 epithelial cells compared to their levels in MG-grown SKOV3 epithelial cells infected with NGgrown S. typhimurium (Chopra et al. 2006).

When mouse macrophages (RAW 264.7) were infected with MG-grown S. typhimurium, activation of mitogenactivated protein kinase kinase 4 (MKK4; a serinethreonine kinase that responds to environmental stimuli) occurred within $2 \mathrm{~h}$ and at a higher level compared with NG-grown bacteria which took $4 \mathrm{~h}$. Interestingly, in a MGgrown RAW 264.7- S. typhimurium co-culture, markedly increased expression of MKK4 was observed (Chopra et al. 2006). Collectively, this evidence suggests that host cells themselves are sensitive to MG and respond to LSMMG exposed bacteria by altering immune response kinetics and magnitude.

Effects of LSMMG in a ground-based system on E. coli and Pseudomonas aeruginosa biofilm formation

Bacteria have been known to colonize diverse ecological habitats where they can exist as free-living cells also known as planktonic cells. Planktonic cells can also exist in a polysaccharide, extra-cellular matrices otherwise known as a biofilms. Biofilm production is controlled by the rpoS gene encoded product $\sigma^{\mathrm{s}}$ (Adams and McLean 1999). Biofilms are a part of the normal growth cycle of most bacteria and are known to cause chronic diseases that are difficult to control or treat. Some of these chronic diseases are endocarditis (infection of the endocardial surface of the heart), cystitis (inflammation of the urinary bladder due to bacterial infection), and bacterial otitis media (inflammation of the middle ear due to bacterial infection).

Within a biofilm, bacteria exhibit greater resistance to a variety of stresses including: oxidative, osmolarity, $\mathrm{pH}$, and antibiotic stresses (Lynch et al. 2006). Moreover, bacterial biofilms can proliferate on board spacecrafts resulting in an unhealthy environment during spaceflight. Also, LSMMG has been shown to induce thicker and more robust biofilm production (Lynch et al. 2006). LSMMG has been known to enhance biofilm resistance to two antibiotics commonly used in treating infectious diseases, chloramphenicol and penicillin. The two aforementioned antibiotics together with osmolarity and ethanol were used as stressors to challenge
MG-exposed biofilm producing E. coli. Each of the four employs a different mode of bacterial cell damage. For example, high salt causes dehydration, ethanol disrupts the cell membrane, penicillin inhibits peptidoglycan synthesis, while chloramphenicol inhibits the synthesis of proteins. In addition to LSMMG inducing thicker $E$. coli biofilms than their NG-grown counterparts, LSMMG-exposed E. coli were also more resistant to the general stressors salt, ethanol, and the two antibiotics penicillin and chloramphenicol (Lynch et al. 2006). Surprisingly, a $\Delta r p o S$ deleted $E$. coli mutant, which produced less extensive biofilms, was compromised in its ability to mount a LSMMG-induced resistance to the general stressors but not to the antibiotics. This evidence suggests that LSMMG-induced resistance to antibiotic challenge is independent of a robust biofilm formation, and that at least two independent pathways of LSMMG-conferred resistance to different types of stresses exist (Lynch et al. 2006).

LSMMG was also used to study biofilm formation in another Gram-negative bacteria, Pseudomonas aeruginosaan opportunistic pathogen. Interestingly, LSMMG induced enhanced biofilm production, similar to what was observed in E. coli. Furthermore, LSMMG appeared to indirectly regulate bacterial virulence through the induction $N$-Acyl homoserine lactone (a quorum-sensing molecule) and its derivatives. In doing so, LSMMG initiates a signaling cascade resulting in a potentially hyper-virulent Psedomonas aerugoinosa (Crabbè et al. 2008).

Taken together, these results highlight the potential increased threat that bacterial biofilms may pose during space exploration.

Effects of LSMMG on gene expression and cell-function and phenotypic response in Saccharomyces cerevisiae eukaryotic yeast cell

Prokaryotic pathogens are not the only pathogen that could be encountered by astronauts in space. The yeast Candidia albicans is part of the oral normal flora. In an immunocompromised host, C. albicans can cause thrush (an opportunistic oral infection) and can cause vulvo-vaginitis in women. As a result, the eukaryotic model organism, $S$. cerevisiae, has been used for MG-related studies. Earlier studies have revealed that during space-like conditions, $S$. cerevisiae experiences different metabolic rates with regards to phosphate uptake (Berry and Volz 1979). More recently, under LSMMG, S. cerevisiae exhibited normal growth rates (with the exception of slightly shortened lag phase) and phenotype with regards to size and shape; however, it did exhibit uncharacteristic random budding along the surface of the cell. Furthermore, using real-time PCR, there was s significant increase in expression of genes associated with budding under MG conditions (Purevdorj- 
Gage et al. 2006). Normally, budding is highly polarized in $S$. cerevisiae and occurs at only one or both ends of the cell. Under MG, the ubiquitous randomly distributed budding could be a result of the LSMMG force affecting the integrity of the cell membrane. Furthermore, using a global transcriptome approach, LSMMG-exposed $S$. cerevisiae differentially expressed 1,372 genes many of which were involved in cell signaling in response to the detection of environmental change (Sheehan et al. 2007). This could play a significant role in the pathogenesis of fungi that become exposed to an MG environment.

The future of space travel and the microbial threat

With the advent of prolonged manned space missions as well as future planned travel to Mars, space microbiology is proving an increasingly relevant subject of study. Eventually, standard air travel will involve planes leaving the Earth's atmosphere and then reentering the atmosphere cutting flight times in less than half of their current lengths. This will, however, expose passengers to SLS as well as the normal flora that they carry in and on them.

With regards to NASA space missions, not only are immuno-compromised astronauts more prone to infection by opportunistic pathogens living under the guise of normal flora but also astronauts could bring back to Earth's inhabitants potentially hyper-virulent bacteria.

Moreover, an interesting observation with regards to MG, virulence, and virulence-associated genes was made. More specifically, despite demonstrating a hyper-virulence phenotype, MG-grown S. typhimurium exhibited decreased levels of TTSS virulence transcripts. S. typhimurium encodes two TTSSs on two independent pathogenicity islands (one is primarily involved with cell invasion while the second is involved with intracellular survival). This counterintuitive finding suggests that MG might negatively regulate TTSS function (while NG growth conditions do not induce TTSS virulence genes) and, that despite doing so, still enhances pathogenesis by some other vague mechanism (Wilson et al. 2002b; Chopra et al. 2006). Interestingly, the virulence associated gene $h f q$ required for optimal virulence of the salmonellae and yersiniae (Sittka et al. 2007; Geng et al. 2009), which encodes the RNA binding and regulatory protein Hfq, was also found to be negatively regulated by actual spaceflight ( 3-fold down-regulated) in S. typhimurium (Wilson et al. 2007). However, other virulence-associated genes involved in RNA metabolism like pnp [encoding polynucleotide phosphorylase/PNPase - an exoribonuclease (Clements et al. 2002; Rosenzweig et al. 2005; Rosenzweig et al. 2007)] and $v a c B$ [encoding RNaseR - another exoribonuclease (Tobe et al. 1992; Erova et al. 2008; Tsao et al. 2009)] were not differentially expressed in S. typhimurium after MG growth.
Unfortunately, MG studies have not included many microorganisms, and other Gram-positive and negative bacteria should be evaluated under MG conditions. For example, the yersiniae are ideal organisms for study on account of $Y$. enterocolitica becoming a leading cause of food-borne illness that could potentially make its way onboard NASA's spacecrafts just as easily as the salmonellae could. Furthermore, Y. pestis has proven itself historically as a formidable pathogen. As a result, it could prove useful to characterize the yersiniae MG response as well as that of other pathogens. In fact, a precedent has already been observed in which salmonellae and yersiniae strains sharing the same gene deletion exhibited entirely opposite affects on bacterial virulence in which the latter was attenuated while the former was enhanced (Clements et al. 2002; Rosenzweig et al. 2005; Rosenzweig et al. 2007). Therefore, previous characterization of the salmonellae and E. coli are not sufficient, and characterization of other MGexposed bacterial pathogens is fully warranted.

Acknowledgements We would like to thank Duane L. Pierson, C. Mark Ott, and Ashok K. Chopra for discussion and guidance in our pursuit of space microbiology knowledge. We would also like to thank Dieter Haas, Shishir Shishodia, and Hector Miranda for valuable feedback and criticism. Work on this manuscript was supported by the National Aeronautics and Space Administration (NASA) cooperative agreement NNX08B4A47A, and Texas Southern University Start-Up Grant Sg0609.

\section{References}

Adams JL, McLean RJ (1999) Impact of rpoS deletion on Escherichia coli biofilms. Appl Environ Microbiol 65:4285-4287

Allen CA, Niesel DW, Torres AG (2008) The effects of low-shear stress on adherent-invasive Escherichia coli. Environ Microbiol 10:1512-1525

Berry D, Volz PA (1979) Phosphate uptake in Saccharomyces cerevisiae Hansen wild type and phenotypes exposed to space flight irradiation. Appl Environ Microbiol 38:751-753

Chopra V, Fadl AA, Sha J, Chopra S, Galindo CL, Chopra AK (2006) Alterations in the virulence potential of enteric pathogens and bacterial-host cell interactions under simulated microgravity conditions. J Toxicol Environ Health A 69:1345-1370

Clements MO, Eriksson S, Thompson A, Lucchini S, Hinton JC, Normark S, Rhen M (2002) Polynucleotide phosphorylase is a global regulator of virulence and persistency in Salmonella enterica. Proc Natl Acad Sci 99:8784-8789

Crabbè A, De Boever P, Van Houdt R, Moors H, Mergeay M, Cornelis $P$ (2008) Use of the rotating wall vessel technology to study the effect of shear stress on growth behaviour of Pseudomonas aeruginosa PA01. Environ Microbiol 10:2098-2110

Erova TE, Kosykh VG, Fadl AA, Sha J, Horneman AJ, Chopra AK (2008) Cold shock exoribonuclease R (VacB) is involved in Aeromonas hydrophila pathogenesis. J Bacteriol 190:3467-3474

Geng J, Song Y, Yang L, Feng Y, Qiu Y, Li G, Guo J, Bi Y, Qu Y, Wang W, Wang X, Guo Z, Yang R, Han Y (2009) Involvement of the post-transcriptional regulator Hfq in Yersinia pestis virulence. PLoS ONE 4:e6213 
Lynch SV, Mukundakrishnan K, Benoit MR, Ayyaswamy PS, Matin A (2006) Escherichia coli biofilms formed under low-shear modeled microgravity in a ground-based system. Appl Environ Microbiol 72:7701-7710

Nickerson CA, Ott CM, Mister SJ, Morrow BJ, Burns-Keliher L, Pierson DL (2000) Microgravity as a novel environmental signal affecting Salmonella enteric serovar Typhimurium virulence. Infect Immun 68:3147-3152

Nickerson CA, Ott CM, Wilson JW, Ramamurthy R, LeBlanc CL, Honer zu Bentrup K, Hammond T, Pierson DL (2003) Low-shear modeled microgravity: a global environmental regulatory signal affecting bacterial gene expression, physiology, and pathogenesis. J Microbiol Methods 54:1-11

Nickerson CA, Ott CM, Wilson JW, Ramamurthy R, Pierson DL (2004) Microbial responses to microgravity and other low-shear environments. Microbiol Mol Rev 68:345-361

Purevdorj-Gage B, Sheehan KB, Hyman LE (2006) Effects of lowshear modeled microgravity on cell function, gene expression, and phenotype in Saccharomyces cerevisiae. Appl Environ Microbiol 72:4569-4575

Rosenzweig JA, Weltman G, Plano GV, Schesser K (2005) Modulation of Yersinia type three secretion system by the S1 domain of polynucleotide phosphorylase. J Biol Chem 280:156-163

Rosenzweig JA, Chromy B, Echeverry A, Yang J, Adkins B, Plano GV, McCutchen-Maloney S, Schesser K (2007) Polynucleotide phosphorylase independently controls virulence factor expression levels and export in Yersinia spp. FEMS Microbiol Lett 270:255-264

Sheehan KB, McInnerney K, Purevdorj-Gage B, Altenburg SD, Hyman LE (2007) Yeast genomic expression patterns in response to low-shear modeled microgravity. BMC Genomics 8:3

Sittka A, Pfeiffer V, Tedin K, Vogel J (2007) The RNA chaperone Hfa is essential for the virulence of Salmonella typhimurium. Mol Microbiol 63:193-217

Tobe T, Sasakawa C, Okada N, Honma Y, Yoshikawa M (1992) vacB, a novel chromosomal gene required for expression of virulence genes on the large plasmid of Shigella flexneri. J Bacteriol 174:6359-6367

Tsao MY, Lin TL, Hsieh PF, Wang JT (2009) The 3'-to-5' exoribonuclease (encoded by HP1248) of Helicobacter pylori regulates motility and apoptosis-inducing genes. J Bacteriol 191:2691-2702

Tucker DL, Ott CM, Huff S, Fofanov Y, Pierson DL, Willson RC, Fox GE (2007) Characterization of Escherichia coli MG1655 grown in a low-shear modeled microgravity environment. BMC Microbiol 7:15

Wilson J, Ott CM, Ramamurthy R, Porwollik S, McClelland M, Pierson DL, Nickerson CA (2002a) Low shear modeled microgravity alters the Salmonella enteric serovar Typhimurium stress response in an RpoS-independent manner. Appl Environ Microbiol 68:5408-5416

Wilson JW, Ramamurthy R, Porwollik S, McClelland M, Hammond T, Allen P, Ott CM, Pierson DL, Nickerson CA (2002b) Microarray analysis identifies Salmonella genes belonging to the low-shear modeled microgravity regulon. Proc Natl Acad Sci USA 99:13807-13812

Wilson JW, Ott CM, Höner zu Bentrup K, Ramamurthy R, Quick L, Porwollik S, Cheng P, McClelland M, Tsaprailis G, Radabaugh T, Hunt A, Fernandez D, Richter E, Shah M, Kilcoyne M, Joshi L, Nelman-Gonzalez M, Hing S, Parra M, Dumars P, Norwood K, Bober R, Devich J, Ruggles A, Goulart C, Rupert M, Stodieck L, Stafford P, Catella L, Schurr MJ, Buchanan K, Morici L, McCracken J, Allen P, Baker-Coleman C, Hammond T, Vogel J, Nelson R, Pierson DL, Stefanyshyn-Piper HM, Nickerson CA (2007) Space flight alters bacterial gene expression and virulence and reveals a role for global regulator Hfq. Proc Natl Acad Sci USA 104:16299-16304

Wilson JW, Ott CM, Quick L, Davis R, Höner zu Bentrup K, Crabbé A, Richter E, Sarker S, Barrila J, Porwollik S, Cheng P, McClelland M, Tsaprailis G, Radabaugh T, Hunt A, Shah M, Nelman-Gonzalez M, Hing S, Parra M, Dumars P, Norwood K, Bober R, Devich J, Ruggles A, CdeBaca A, Narayan S, Benjamin J, Goulart C, Rupert M, Catella L, Schurr MJ, Buchanan K, Morici L, McCracken J, Porter MD, Pierson DL, Smith SM, Mergeay M, Leys N, Stefanyshyn-Piper HM, Gorie D, Nickerson CA (2008) Media ion composition controls regulatory and virulence response of Salmonella in spaceflight. PLoS ONE 3:e3923 